\title{
Examination of the Reproducibility of Grip Force and Muscle Oxygenation Kinetics on Maximal Repeated Rhythmic Grip Exertion
}

\author{
Masakatsu Nakada $^{1)}$, Shinichi Demura ${ }^{2)}$, Shunsuke Yamaji ${ }^{3)}$, and Yoshinori Nagasawa ${ }^{4)}$ \\ 1) National Defence of Academy \\ 2) Department of Physical Education, Kanazawa University \\ 3) Fukui National College of Technology \\ 4) Akita Prefectural University
}

\begin{abstract}
The contribution of physiological mechanisms involving force-exertion value during maximal repeated rhythmic muscle contraction work changes over time. The purpose of this study was to examine the reproducibility of grip force and muscle oxygenation kinetics with a decrease of the gripping force during maximal repeated rhythmic grip (RRG). Subjects were 10 males, aged 20-26 years (height $173.9 \pm 7.3 \mathrm{~cm}$, body weight $71.5 \pm 11.2 \mathrm{~kg}$ ). Each subject performed maximal repeated rhythmic grip as a target value with a target frequency of $30 \mathrm{grips} \cdot \mathrm{min}^{-1}$ for $6 \mathrm{~min}$. The trialto-trial reproducibility of Oxygenated haemoglobin $(\mathrm{Oxy}-\mathrm{Hb})$, Deoxygenated haemoglobin (Deoxy-Hb), Total haemoglobin (Total $\mathrm{Hb}$ ) and grip force during the RRG (6 min) was very high $\left(\mathrm{r}_{\mathrm{xy}}=0.919-0.966\right)$ and the decreasing pattern of the force-time curve was consistent. The cross correlation coefficients of the grip force $\left(r_{x y}=0.985\right)$ and muscle oxygenation kinetics (Total Hb: 0.996, Oxy-Hb: 0.992, Deoxy$\mathrm{Hb}:$ 0.995) in the pre-inflection phase (marked force decreasing phase) were very high, while these coefficients in the post-inflection phase (almost steady state phase) were low as compared with those in the pre-inflection phase. The trialto-trial reliabilities of any parameter regarding grip were fair or high $(\mathrm{ICC}=0.686-0.927)$. The changing points of muscle oxygenation kinetics appeared before reaching an almost steady state, which showed a high reliability and they were considered to reflect the shift of physiological mechanisms. In particular, the intraclass correlation coefficients (ICC) for the time to reach maximum Deoxy-Hb and Oxy-Hb values and regression coefficient in an increasing phase of $\mathrm{Oxy}-\mathrm{Hb}$ were very high $(\mathrm{ICC}=0.894-0.947)$. It was found that the trial-totrial reproducibility of grip force and muscle oxygenation kinetics is very high during the whole $6 \mathrm{~min}$ in RRG, but is poor during the post-inflection phase. The reproducibility of the grip force and muscle oxygenation kinetics in the phase before reaching an almost steady state during RRG is fair, and the decrease of the grip force in this phase may be influenced by the muscle oxygenation kinetics. J Physiol Anthropol Appl
\end{abstract}

Human 24 (1): 1-6, 2005 http://www.jstage.jst.go.jp/browse/jpa

Keywords: near infrared spectroscopy, repeated rhythmic grip, force-time curve, inflection time, muscle oxygenation kinetics

\section{Introduction}

Muscle endurance, generally, has been quantitatively evaluated by time-series digital data during repeated rhythmic or sustained static muscle contraction (Bemben et al., 1996; Clarke et al., 1992; Yamaji et al., 2000). It takes a relatively long time to measure this as compared with that of maximal strength and muscle power. Prolonged muscle contraction imposes a high pain on the subjects, and an exertion value involves more in the way of measurement errors (Bowie and Cumming, 1971; Nagasawa et al., 2000). Measurement of muscle endurance is apt to have lower reproducibility than that of maximal strength and muscle power. The reproducibility of a force-time curve during sustained muscle contraction has been examined (Capodaglio et al., 1997; Lagerstorm and Nordgren, 1996; Lagerstrom and Nordgren, 1998; Wallstrom and Nordenskiold, 2001), but only for parameters to evaluate a part of the force-time curve (e.g. the integrated area and sustaining time). In addition, the parameters differed in each study. A decrease of sustained muscle contraction does not follow a straight line. Two or three different phases of decreasing speed appear because the contribution of the physiological mechanisms (e.g., muscle oxygenation, recruitment of muscle fibre) involving maximal repeated rhythmic muscle contractions changes over time. The reproducibility of sustained muscle contraction in each decreasing speed phase is not considered to be the same because of the different contribution of physiological mechanisms. When evaluating muscle endurance from sustained muscle contraction, selecting the phase with low 
reproducibility is a problem. Therefore, the reproducibility of each decreasing phase of force-exertion or the changing phase of physiological mechanisms related to sustained muscle contraction need to be clarified. There are few examinations of the reproducibility of physiological mechanisms related to muscle contraction.

In repeated rhythmic muscle contraction with a high load, muscle blood flow kinetics differs in the initial and latter phases. In the former phase, a blood flow obstruction occurs by an increase in intra-muscular pressure, and in the latter phase there is a resumption of blood flow (Humphreys and Lind, 1967; Nielson and Ingvar, 1967). The decreasing phase of the force-exertion value can be divided into two phases that show a marked decrease (the initial phase) and an almost steady state (the latter phase) (see Fig. 1). These two phases depend on different physiological mechanisms (Yamaji et al., 2000).

The purpose of this study was to examine the reproducibility of grip force and muscle oxygenation kinetics with a decrease of gripping force during maximal repeated rhythmic grip (RRG).

\section{Methods}

\section{Subjects}

Subjects were 10 healthy males without upper extremity impairments, aged 20-26 years (height $173.9 \pm 7.3 \mathrm{~cm}$, body weight $71.5 \pm 11.2 \mathrm{~kg}$ ). Their physiques approximated standard values for Japanese males of the same age-stage (Lab Physical Edu in Tokyo Met Univ 2000). Written informed consent was obtained from all subjects after a full explanation of the experimental purpose and protocol.

\section{Materials}

Grip strength was measured using a digital hand dynamometer with a load-cell sensor (EG-100, SAKAI, Japan). Each signal during RRG was sampled at $20 \mathrm{~Hz}$ with an analogue-to-digital interface and then relayed to a personal computer. To increase the motivation of the subjects during RRG, the input digital data was immediately displayed on a screen as a force-time curve to give feedback. Near infrared spectroscopic (NIRS) instrument (PSA-IIIN, Biomedical Science, Japan) was used to evaluate the muscle oxygenation of the forearm during RRG. A PSA-IIIN using threewavelengths and two optical detectors analysed absorbance of three-wavelengths based on the Lambert-Beer law, and the measured tissue oxygen saturation $\left(\mathrm{StO}_{2}\right)$ and total tissue haemoglobin (Total $\mathrm{Hb}$ ). The probe contained a light source that was filtered at 700,750 and $830 \mathrm{~nm}$. The two optical detectors were placed $15 \mathrm{~mm}$ and $25 \mathrm{~mm}$, respectively, from the light source. Transmitted light from the probe was then either absorbed or scattered within the tissue. Scattered light was delivered via two fibre-optic light detectors of the same size to a photomultiplier every $0.1 \mathrm{sec}$. The probe was engineered for a photon depth penetration of $15-25 \mathrm{~mm}$. A previous paper by Sakai and Saito (1995) discussed the principle of a PSA-IIIN in detail.

\section{Experimental procedure}

Each subject's dominant hand was judged using Oldfield's handedness inventory (Oldfield, 1971). All subjects performed the handgrip test with the dominant hand while seated in an adjustable ergometric chair. The arm was in a sagittal and horizontal position and was supported by an armrest with the forearm vertical and the hand in a semi-prone position. The grip width was individually adjusted to achieve a 90-degree angle with the proximal-middle phalanges. After measuring the maximal grip force, each subject performed the RRG test using maximal grip force as a target value with a target frequency of $30 \mathrm{grips} \cdot \mathrm{min}^{-1}$ for $6 \mathrm{~min}$. Namely, the subjects grasped with a maximal effort when a beep sound uttered from the instrument at intervals of 2 seconds for 6 min (180 times), and peaks exerted during the interval were recorded. No verbal

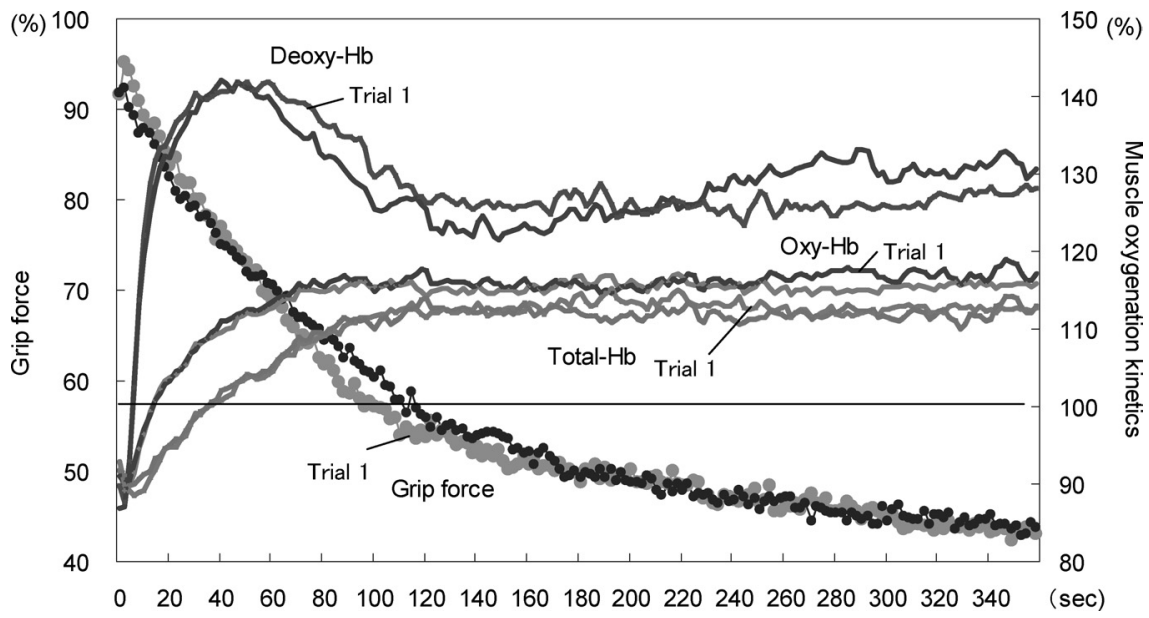

Fig. 1 Mean curves of Total-Hb, Oxy-Hb, Deoxy-Hb, and grip force for two trials. Right axis indicates the scales of relative muscle oxygenation kinetics value (\%) based on resting value. Left axis indicates the scales of relative grip force value (\%) based on maximal grip force. 
encouragement was given during the test. A NIRS probe was positioned over a surface of the flexor digitorum superficialis of the dominant hand and muscle oxygenation kinetics were continuously monitored during a rest for $2 \mathrm{~min}$ and a RRG test after a rest for $10 \mathrm{~min}$. To examine the reproducibility of muscle oxygenation kinetics, subjects re-performed RRG by the above-stated experimental procedure on a different day. Before the second trial we confirmed that $\mathrm{StO}_{2}$ and Total $\mathrm{Hb}$ were stable at almost the same value as the first trial and the probe was positioned roughly over the same position.

\section{Force-time parameters}

The force-time curve was obtained by plotting the peak force from every RRG, namely 180 grips $=30$ grips $\cdot \min ^{-1} \times 6 \mathrm{~min}$. The grip force during $\mathrm{RRG}$ was converted to a relative value based on the maximal grip force value for individuals, and the relative value was used for data analysis. In this study, the inflection point of the decreasing speed of force-time curve was calculated statistically to distinguish between a marked decreasing phase and an almost steady state phase. Many researchers (Kurpad et al., 2001; Lee et al., 1990; Soler et al., 1989) have proposed a method to examine the inflection point (break point) statistically by applying a two-phase regression model to time series data that can assume two phases. The inflection point was the time at which the decreasing speed of force exertion remarkably changed, and it was calculated statistically from two regression lines fitted to each decreasing phase by applying this model. The decrease of the force exertion value in the pre-inflection phase was marked, while in the post-inflection phase it was almost at a steady state.

The inflection point (time) was determined by the following conditions:

1) The time series force-time data (180 data) divided into the former and latter phases at all combinations (e.g. the former: the latter, $3: 177,4: 176, \ldots, 176: 4,177: 3)$, and the respective regression lines were calculated.

2) The best fit regression lines were determined by the following conditions: the regression coefficients (a1) in the pre-inflection phase were more significant and greater than the regression coefficients (a2) in any other post-inflection phase, and the sum of the determination coefficients of both regression equations was the highest.

3) The inflection point was determined at the time from the best fit regression lines in the combination of time series data.

The parameters to evaluate the force-time curve were selected from the time of decreasing 20,30, and 40\% from the maximal grip, the mean peak force value (sum of peak force in each exertion/180 times), and the rate of decrease constant $(\mathrm{k})$ in the exponential function $\left(y=a^{e^{-k t}}+b\right)$.

\section{Muscle oxygenation kinetics}

Muscle oxygenation kinetics during RRG, with oxygenated, deoxygenated and total haemoglobins (Oxy-Hb, Deoxy-Hb, and Total $\mathrm{Hb}$, respectively) as parameters, was examined using the following equations;

$$
\begin{aligned}
& \mathrm{Oxy}-\mathrm{Hb}=\text { Total } \mathrm{Hb} \times \mathrm{StO}_{2} \\
& \text { Deoxy-Hb }=\text { Total } \mathrm{Hb}-\mathrm{Oxy}-\mathrm{Hb}
\end{aligned}
$$

The data obtained by NIRS was converted to relative values based on each individual average value that was measured during resting on an armrest at the heart level for $2 \mathrm{~min}$. The change of Oxy-Hb was evaluated at the time of reaching a minimum or maximum value, and the regression coefficient in the remarkably increasing phase. The change of Deoxy-Hb was evaluated at the time of reaching a maximal value (the onset of a decrease) and a steady state value, and the regression coefficient in the remarkably decreasing phase until reaching a steady state value. The regression coefficient during the change of Oxy-Hb and Deoxy-Hb was calculated according to the above inflection model.

\section{Data analysis}

Time-series data of the force and muscle oxygenation during RRG for 6 min was averaged for all data samples for all subjects, and the cross correlation coefficient between two trial values was calculated for the whole $6 \mathrm{~min}$, the pre-inflection phase, and the post-inflection phase. The reliability of parameters regarding the force-time value and a changing point of the muscle oxygenation was examined by intra-class correlation coefficients (ICC). The probability level of 0.05 was an indication of the statistical significance.

\section{Results}

\section{Reproducibility of grip force and muscle oxygenation kinetics during RRG}

The cross correlation coefficients of grip force and muscle oxygenation kinetics (Total $\mathrm{Hb}, \mathrm{Oxy}-\mathrm{Hb}$, and Deoxy-Hb) between trials were very high $\left(r_{x y}=0.884-0.994\right)$ (see Table 1). Although the coefficients of these time-series values in the preinflection phase (remarkably force decreasing initial phase) were also very high $\left(\mathrm{r}_{\mathrm{xy}}=0.985-0.996\right)$, coefficients in the post-inflection phase (almost steady state phase) tended to be lower than those in the pre-inflection phase. In particular, the coefficients of Total $\mathrm{Hb}$ and Deoxy-Hb were very low $\left(r_{x y}=0.378,0.262\right.$, respectively $)$. All coefficients were obtained

Table 1 The trial-to-trial cross correlation coefficients of grip force and muscle oxygenation kinetics

\begin{tabular}{lccc}
\hline & Whole 6 min & $\begin{array}{c}\text { Pre-inflection } \\
\text { phase }\end{array}$ & $\begin{array}{c}\text { Post-inflection } \\
\text { phase }\end{array}$ \\
\hline Grip force & 0.994 & 0.985 & 0.978 \\
Total Hb & 0.987 & 0.996 & 0.378 \\
Oxy-Hb & 0.988 & 0.992 & 0.716 \\
Deoxy-Hb & 0.884 & 0.995 & 0.262 \\
\hline
\end{tabular}

Note: All coefficients were obtained with a 0 time lag. 
Table 2 Intraclass correlation coefficients (ICC) of force-time parameters muscle oxygen kinetics parameters

\begin{tabular}{|c|c|c|c|c|c|c|c|}
\hline & & \multicolumn{2}{|c|}{ Trial 1} & \multicolumn{2}{|c|}{ Trial 2} & \multirow[b]{2}{*}{$t$} & \multirow[b]{2}{*}{ ICC } \\
\hline & & mean & $\mathrm{SD}$ & mean & SD & & \\
\hline \multicolumn{8}{|l|}{ Muscle oxygen kinetics parameters } \\
\hline Time to reach a minimum Oxy-Hb value & $(\mathrm{sec})$ & 9.8 & 5.4 & 6.7 & 4.1 & 2.055 & 0.625 \\
\hline Time to reach maximum $\mathrm{Oxy}-\mathrm{Hb}$ value & $(\mathrm{sec})$ & 132.8 & 59.0 & 140.5 & 61.2 & -0.847 & 0.892 \\
\hline Time to reach maximum Deoxy-Hb value & $(\mathrm{sec})$ & 34.8 & 12.3 & 40.4 & 20.5 & -0.966 & 0.892 \\
\hline Regression coefficient in the decreasing phase of Deoxy-Hb & & -0.3 & 0.2 & -0.4 & 0.4 & 1.018 & 0.912 \\
\hline Regression coefficient in the increasing phase of $\mathrm{Oxy}-\mathrm{Hb}$ & & 0.5 & 0.4 & 0.7 & 0.5 & -1.906 & 0.845 \\
\hline \multicolumn{8}{|l|}{ Force decreasing parameters } \\
\hline Inflection time & $(\mathrm{sec})$ & 68.6 & 13.9 & 75.7 & 24.5 & -1.107 & 0.623 \\
\hline Rate of decrement constant ( $\mathrm{k}$ ) in the exponential function & & -2.6 & 1.5 & -2.3 & 0.9 & -0.690 & 0.578 \\
\hline Time decreasing $20 \%$ from maximal grip & $(\mathrm{sec})$ & 35.4 & 15.0 & 34.2 & 15.9 & 0.431 & 0.587 \\
\hline Time decreasing $30 \%$ from maximal grip & $(\mathrm{sec})$ & 58.2 & 18.7 & 61.8 & 17.2 & -0.668 & 0.597 \\
\hline Time decreasing $40 \%$ from maximal grip & $(\mathrm{sec})$ & 105.6 & 44.7 & 111.4 & 36.1 & -0.583 & 0.747 \\
\hline Mean grip force for 30 seconds & $(\mathrm{kg})$ & 54.6 & 6.9 & 55.1 & 5.6 & -0.448 & 0.874 \\
\hline
\end{tabular}

with a 0 time lag.

\section{Reliability of parameters regarding the force-time curve} and the changing point of muscle oxygenation kinetics during $R R G$

It is necessary to clarify the time-series variance patterns of muscle oxygenation because the reliability was examined at the changing points of each parameter. Figure 1 shows the average curves of changes for Oxy-Hb, Deoxy- $\mathrm{Hb}$, Total $\mathrm{Hb}$ and grip force during the RRG for 6 min. All subjects in this study showed almost the same pattern as shown in Figure 1. Total $\mathrm{Hb}$ and $\mathrm{Oxy}-\mathrm{Hb}$ decreased linearly for $4-10 \mathrm{sec}$ after the onset of RRG, and then increased remarkably for about 100 sec. After that, they reached an almost steady state with a higher level than that at rest (about 110-115\%). Deoxy-Hb increased remarkably for about $50 \mathrm{sec}$, and then decreased rapidly for about $100 \mathrm{sec}$, followed by an almost steady state with a higher level than that at rest (about 130-140\%).

Table 2 shows the intraclass correlation coefficients (ICC) of force-time parameters and muscle oxygen kinetics parameters. The ICC of the time of reaching a maximal value for Oxy-Hb and Deoxy-Hb was very high ( $\mathrm{ICC}=0.894,0.892$, respectively), while the ICC of the time of reaching a minimum value for $\mathrm{Oxy}-\mathrm{Hb}$ was moderate $(\mathrm{ICC}=0.625)$. Furthermore, the ICC of the regression coefficient during an increasing phase for $\mathrm{Oxy}-\mathrm{Hb}$ and a decreasing phase for Deoxy-Hb was very high ( $\mathrm{ICC}=0.912,0.845$, respectively). The ICC of the force-time parameters was moderate or higher $(\mathrm{ICC}=0.578-0.874)$.

\section{Discussion}

Muscle endurance has been evaluated by the repeated rhythmic or sustained static muscle contraction performed with relative load intensities based on an individual's maximal strength. In previous studies, the muscle endurance has been estimated by sustained muscle contraction (e.g. handgripping, elbow flexion) for $1-12 \mathrm{~min}$ or until being completely exhausted with relative load intensities (Bemben et al., 1996; Clarke et al., 1992; Yamaji et al., 2000; Walamies and Tunjanmaa, 1993). It is possible that the measurement of muscle endurance, which has a longer measurement time as compared with a test of the maximal strength or muscle power, is affected by the psychological factor of the suffering pain with the muscle fatigue (Bowie and Cumming, 1971; Nagasawa et al., 2000). Walamies and Tujanmaa (1993) pointed out that the decrease in motivation caused by the long measurement time would affect the force-exertion value. A sustained or repeated force-exertion value is considered to involve a larger measurement error (Yamaji et al., 2002). Moreover, the reproducibility of repeated force-exertion value may differ in each decreasing phase, because the contribution of the physiological mechanisms related to muscle contraction changes with the working time. When evaluating muscle endurance it is important to select the decreasing phases with high reproducibility in which physiological mechanisms related to muscle contraction are considered.

There are some studies examining the reproducibility of force-time parameters during sustained muscle contraction (Capodaglio et al., 1997; Lagerstorm and Nordgren, 1996; Lagerstrom and Nordgren, 1998; Wallstrom and Nordenskiold, 2001). It was reported that the reproducibility of the force-time parameters (mean peak force and the rate of decrease constant (k) in the exponential function) selected in these studies was high (Lagerstorm and Nordgren, 1996; Wallstrom and Nordenskiold, 2001), consistent with the results of the present study. The parameters reflect the whole tendency of force decreasing. However, there are few studies that examined the reproducibility of the time-series force decreasing and muscle oxygenation kinetics during sustained muscle contraction.

Yamaji et al., (2004) reported that the changing point for a decreased speed of gripping force (inflection point) during the sustained isometric gripping reflects the changing point for muscle oxygenation kinetics, and the contribution of physiological mechanisms related to the gripping may differ at the phase before/after the inflection point. The reliability of the 
inflection time calculated in the present study $(\mathrm{ICC}=0.623)$ was similar to other force-time parameters (Bemben et al., 1996; Bowie and Cumming, 1971; Nagasawa et al., 2000; Walamies and Tunjanmaa, 1993) and the parameters regarding muscle oxygenation kinetics used in previous studies (Yamaji et al., 2000, 2004). Therefore, it is valid to examine the reproducibility of the force-time curve by dividing into preand post-inflection points.

Boushel et al. (1998) reported that muscle oxygenation kinetics of the forearm flexor muscle by NIRS closely reflected the exercise intensity during the rhythmic handgripping. From the present results, it is judged that the trial-to-trial similarity of these parameters is very high for the whole $6 \mathrm{~min}$. In examining the two different phases based on the inflection point of the decreasing the grip force speed, the post-inflection phase tended to show lower reproducibility of grip force and muscle oxygenation kinetics than the pre-inflection phase. The sustained grip force in the initial phase before the inflection point is affected by the blood flow obstruction caused by an increase in intra-muscular pressure (Yamaji et al., 2004). The latter phase after the inflection point occurs with an increase in the oxygen supply caused by the resumption of blood flow with the a decrease of the intra-muscular pressure, and actively promotes the rotation of the recruitment of muscle fibre unit with muscle fatigue to maintain the muscle contraction level (Clarke et al., 1992; Gerdle and Fugl-Meyer, 1992; St Clair Gibson et al., 2001). In time-series variance, a small measurement error in the initial phase becomes a greater error in the latter phase because each measured value depends on the variance of the former measured value (Jones, 1985). In addition, the effect of the psychological factor of a pain with muscle fatigue may cause a large measurement error in the latter phase (Bowie and Cumming, 1971; Nagasawa et al., 2000). Accordingly, the reproducibility in the latter phase may become lower. The reproducibility of muscle oxygenation kinetics in the latter phase was remarkably decreased as compared with that of grip force. In the latter phase, the griping value and muscle oxygenation kinetics reached an almost steady state. In this phase, since the muscle unit rotation as stated above and a subtle change of the grip motion (e.g. wrist joint angle) occur to compensate for a decreasing force from muscle fatigue, the contribution of each flexor muscle related to grip motion rapidly changes (St Clair Gibson et al., 2001). The lower reproducibility of muscle oxygenation kinetics in this phase may be due to the contribution difference between trials.

Yamaji et al. (2000) reported that the reproducibility of grip force and the muscle oxygenation kinetics during the maximal sustained isometric gripping was very high. On the one hand, in the contraction, because muscle contraction is maintained during the measurement, the obstruction of muscle blood flow in the initial phase may also be maintained. On the other hand, since the repeated rhythmic exercise repeats muscle contraction and relaxation by regulated exertion frequency (this study: 1 time at $2 \mathrm{sec}$ ), the variance of muscle blood flow and oxygenation kinetics around the exertion muscles also become greater. Therefore, the reproducibility of muscle oxygenation kinetics during the repeated exercise and sustained isometric exercise may be lower.

Total $\mathrm{Hb}$ and $\mathrm{Oxy}-\mathrm{Hb}$ were remarkably decreased after starting RRG. The time of reaching the minimum value of $\mathrm{Oxy}-\mathrm{Hb}$ reflects the phase of the blood flow obstruction caused by an increase of intra-muscular pressure due to a high contraction level (Humphreys and Lind, 1963; Yamaji et al., 2004). The reliability of these parameters is good. Moreover, the reproducibility of the phase, which decreased $10 \%$ from the maximal grip, is fair $(\mathrm{ICC}=0.625)$. Deoxy-Hb was increased remarkably for about $50 \mathrm{sec}$. The time of reaching the maximal Deoxy-Hb value reflects the phase where Deoxy$\mathrm{Hb}$ increased without being eliminated due to the influence of the blood flow obstruction (Clarke et al., 1992; Kahn et al., 1998). The phase after the time of reaching the maximal Deoxy-Hb value corresponds to the perfusion volume exceeding the increasing volume of Deoxy-Hb because of the resumption of blood flow. The grip force at this phase decreased to near the inflection point, namely until decreasing about $30 \%$ from the maximal grip. These parameters (inflection time and time decreasing $30 \%$ from maximal grip) showed good reliability (ICC $=0.623,0.597$, respectively), and were similar to the time of reaching the maximal Deoxy- $\mathrm{Hb}$ value. The inflection point is considered to correspond to the phase from the obstruction of blood flow to the beginning of resumption, supporting the hypothesis that it reflects not only the changing point of the speed of the force decrease, but also the change of the physiological mechanisms (Yamaji et al., $2000,2004)$. It would be useful to divide this into two phases.

The regression coefficient of the Deoxy-Hb decreasing phase reflects the phase from the beginning of the decrease of Deoxy-Hb to the balance of Deoxy-Hb production and its elimination (Clarke et al., 1992; Kahn et al., 1998). The grip force in this phase decreased $40 \%$ from the maximal grip. Moreover, the time of reaching maximal $\mathrm{Oxy}-\mathrm{Hb}$ value reflected the phase corresponding to the oxygen demand and supply to muscle, and the regression coefficient of the $\mathrm{Oxy}-\mathrm{Hb}$ increasing phase reflected from the beginning of the resumption of blood flow to the above-stated balanced phase. The grip force decreased at an almost steady state (decreasing $40-60 \%$ from maximal grip) in this phase. These muscle oxygenation parameters and the time of decreasing $40 \%$ from the maximal grip showed good reliability (ICC $=0.845-0.912$ and 0.747 , respectively). Accordingly, the reproducibility of grip force and muscle oxygenation kinetics in the phase until reaching a steady state would be good (grip force: ICC= 0.985 , oxygenation kinetics: ICC $=0.992-0.996$ ). However, the reproducibility after this phase may be poor, since the postinflection phase tended to show lower reliability than the preinflection phase (oxygenation kinetics: ICC $=0.262-0.716$ ). Although the individual difference of all muscle oxygenation parameters was larger than that of force-time parameters (Table 2), the intra-subject variance of these parameters is 
small, and the reliability of these parameters is as good as that of the force-time parameters.

In conclusion, the reproducibility of the grip force and muscle oxygenation kinetics during the whole $6 \mathrm{~min}$ is very good. The post-inflection phase tended to show poor reproducibility. Therefore, it was judged that the reproducibility of the phase until reaching the steady state would be fair. The decreasing grip force would correspond to muscle oxygenation kinetics, and it is possible that the evaluating phase for muscle endurance by considering physiological mechanisms related to the muscle contraction can be established from the decreasing tendency of the grip force.

\section{References}

Bemben MG, Massey BH, Bemben DA, Misner JE, Boileau RA (1996) Isometric intermittent endurance of four muscle groups in men aged 20-74 yr. Med Sci Sports Exerc 28: 145-154

Boushel R, Pott F, Madsen P (1998) Muscle metabolism from near infrared spectroscopy during rhythmic handgrip in humans. Eur J Appl Physiol 79: 41-48

Bowie W, Cumming GR (1971) Sustained handgripreproducibility; effect of hypoxia. Medicine and Science in Sports 3: 24-31

Capodaglio P, Maestri R, Bazzini G (1997) Reliability of a handgripping endurance test. Ergonomics 40: 428-34

Clarke DH, Molly QH, Dotson CO (1992) Muscular strength and endurance as a function of age activity level. Res Q Exerc Sport 63: 302-310

Gerdle B, Fugl-Meyer AR (1992) Is the mean power frequency shift of the EMG a selective indicator of fatigue of the fast twitch motor units? Acta Physiol Scand145: 129-38

Hermansen L, Hultman E, Saltin B (1967) Muscle glycogen during prolonged severe exercise. Acta Physiol Scand 71: 129-139

Humphreys PW, Lind AR (1963) The blood flow through active and inactive muscle of the forearm during sustained handgrip contraction. J Physiol 166: 120-135

Jones RH (1985) Repeated measures, interventions, and time series analysis. Psychoneuroendocrinology 10: 5-14

Kahn JF, Jouanin JC, Bussiere JL, Tinet E, Avrillier S, Ollivier JP, Monod H (1998) The isometric force that induces maximal surface muscle deoxygenation. Eur J Appl Physiol 78: $183-187$

Kurpad AV, Raj T, El-Khoury A, Beaumier L, Kuriyan R, Srivatsa A, Borgonha S, Selvaraj A, Regan MM, Young VR (2001) Lysine requirements of healthy adult Indian subjects, measured by an indicator amino acid balance technique. Am J Clin Nutr 73: 900-907

Lab Physical Edu in Tokyo Met Univ (ed) (2000) Physique. New physical fitness standards of Japanese people. Fumaido, Tokyo

Lagerstrom C, Nordgren B (1996) Methods for measuring maximal isometric grip strength during short and sustained contractions, including intra-rater reliability. Ups J Med Sci 101: 273-285

Lagerstrom C, Nordgren B (1998) On the reliability and usefulness of methods for grip strength measurement. Scand J Rehabil Med 30: 113-119

Lee ML, Poon WY, Kingdon HS (1990) A two-phase linear regression model for biologic half-life data. J Lab Clin Med 115: 745-748

Nagasawa Y, Demura S, Yoshimura Y Yamaji S, Nakada M, Matsuzawa J (2000) Relationship between strength exertion and subjective muscle-farigue sensation in the relative sustained static hand gripping. Jpn J Phys Fitness Sports Med 49: 495-502

Nielson B, Ingvar D (1967) Intramuscular pressure and contractile strength related to muscle blood flow in man. Scand J Clin Lab Invest 99: 31-38

Oldfield RC (1971) The assessment and analysis of handedness: the Edinburgh inventory. Neuropsychologia 9: 97-113

Sakai A, Saito T (1995) Noninvasive measurement of tissue oxygen consumption in human using near-infrared spectroscopy. Therapeutic Res 16: 247-250 [In Japanese]

Soler AM, Folledo M, Martins LE (1989) Anaerobic threshold estimation by statistical modelling. Braz J Med Biol Res 22: 795-797

St Clair Gibson A, Lambert ML, Noakes TD (2001) Neural control of force output during maximal and submaximal exercise. Sports Med 31: 637-50

Walamies M, Tunjanmaa V (1993) Assessment of the reproducibility of strength and endurance handgrip parameters using a digital analyser. Eur J Appl Physiol 67: 83-86

Wallstrom A, Nordenskiold U (2001) Assessing hand grip endurance with repetitive maximal isometric contractions. J Hand Ther 14: 279-85

Yamaji S, Demura S, Nagasawa Y Nakada M, Yoshimura Y, Matsuzawa J, Toyoshima Y (2000) Examination of the parameters of static muscle endurance on sustained static maximal hand gripping. Japan J Phys Educ 45: 695-706

Yamaji S, Demura S, Nagasawa Y, Nakada M, Kitabayashi T (2002) The effect of measurement time when evaluating static muscle endurance during sustained static maximal gripping. J Physiol Anthropol Appl Human Sci 21: 151-158

Yamaji S, Demura S, Nagasawa Y, Nakada M (2004) Relationships between decreasing force and muscle oxygenation kinetics during sustained static gripping. J Physiol Anthropol Appl Human Sci 23: 41-47

Received: June 28, 2004

Accepted: October 7, 2004

Correspondence to:Shunsuke Yamaji, Fukui National College of Technology, Geshi, Sabae, Fukui 916-8507, Japan

Phone: +81-778-62-8229

Fax: +81-778-62-3417

e-mail: yamaji@fukui-nct.ac.jp 